Alvin B. Javierto, MD

Josefino G. Hernandez, MD

Rodante A. Roldan, MD

Department of Otolaryngology

Head and Neck Surgery

Rizal Medical Center

\section{Foreign Body in the Sphenoid Sinus}

\section{Dear Editor,}

Foreign bodies in the paranasal sinuses are not so common but are still possible. The structures most often involved are maxillary and the frontal sinuses.' In our case, the sphenoid sinus which is posterior and deep was involved. Having a foreign body lodged in the sphenoid sinus, and considering how it got there put the patient at great risk of possible involvement of the optic nerve and the carotid artery. Accessing the sphenoid sinus and removing the foreign body lodged in it would be a big challenge to any surgeon. We report one such case.

\section{CASE REPORT}

A 22-year-old man was accidentaly shot in the face by a fellow criminology student while playing with a polyvinyl chloride (PVC) handmade gun two weeks prior to admission. The patient, who was conscious, coherent and ambulatory at that time, was brought to a local government hospital where facial CT scans revealed a radio-opaque, well rounded foreign body, approximately measuring $1.5 \mathrm{~cm} \times 1.5 \mathrm{~cm}$ in diameter lodged in the sphenoid sinus. (Figure $1 \mathrm{~A} \& B$ )
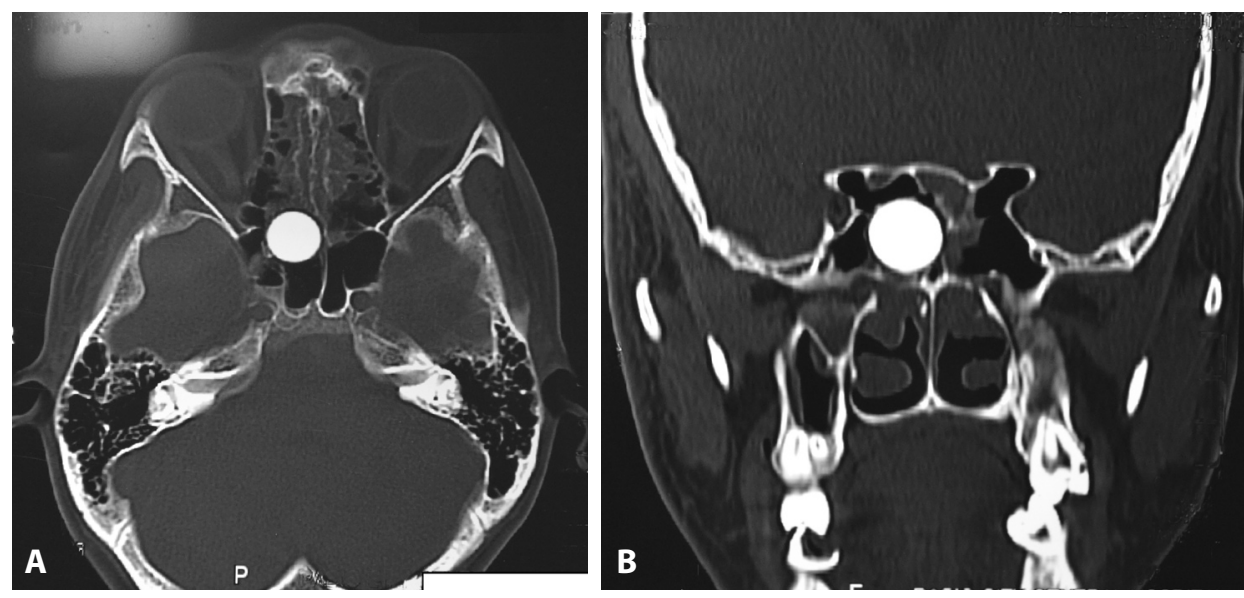

Figures 1A. Axial CT showing a radio-opaque foreign body at the level of the right sphenoid sinus; B. Coronal CT showing a radio-opaque foreign body in the right sphenoid sinus 


\section{LETTERS TO THE EDITOR}

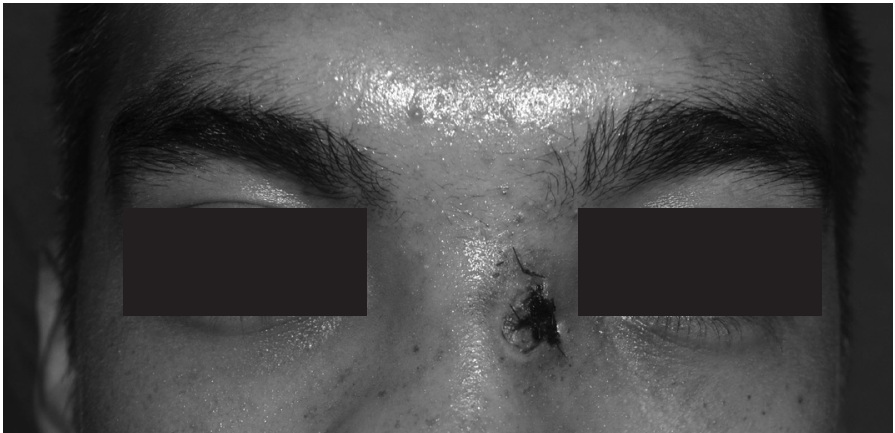

Figure 2 Sutured point of entry with scab, left lateral nasal root

He was subsequently admitted on Penicillin $G$ and was eventually discharged. On his fourth post-injury day, he had profuse epistaxis from the right nostril and consulted at the emergency room of our medical center. Anterior nasal packing did not control the bleeding and was converted to a posterior nasal pack. A sutured wound with a scab on the left lateral nasal root was also noted. (Figure 2) The rhinology service consultants advised endoscopic removal of the foreign body under general anesthesia. Intraoperatively, the nasal cavity was congested with slight septal deviation to the right and a collapsed posterosuperior septal wall. Behind the postero-superior third segment of the middle meatus, the sphenoethmoidal recess was appreciated. On further exploration, a $1.5 \mathrm{~cm}$ x $1.5 \mathrm{~cm}$ green marble was seen lodged in the sphenoid sinus. An initial attempt to remove the foreign body using a nasal foreign body extractor failed. An improvised large metallic paper clip, molded to the shape of a curved foreign body extractor was also unsuccessful. A cotton pledget tip dipped in cyanoacrylate (super glue) also failed to have the marble attach to it. Two angulated sharp foreign body extractors insinuated using the four hand technique yet again failed. The collapsed posterior end of the nasal septum was removed using a cutting forceps for better visualization and access and on the last attempt, a bent spoon used to scoop the marble out of the sphenoid sinus was successful. (Figure 3) Full extraction of the foreign body was achieved by dislodging the marble towards the nasopharynx and into the oral cavity without compromising the optic nerve and the carotid artery. (Figure 4)

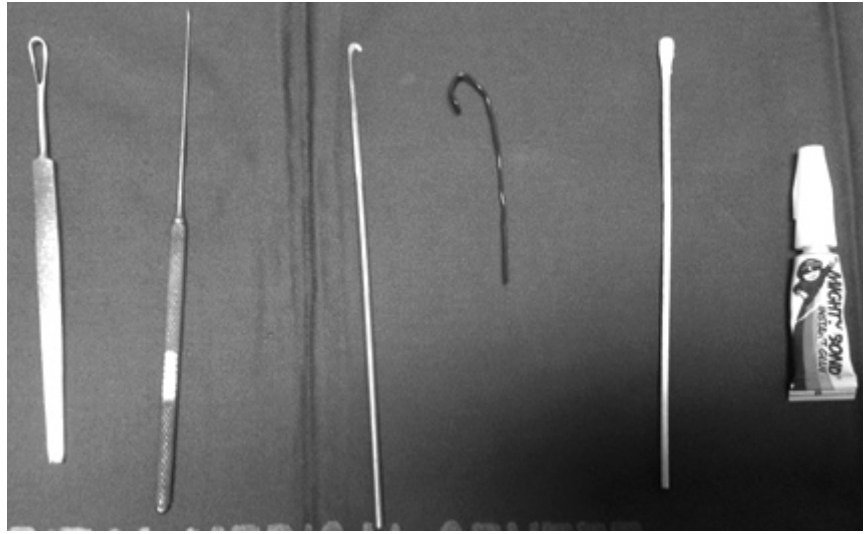

Figure 3. Instruments used during the removal of foreign body

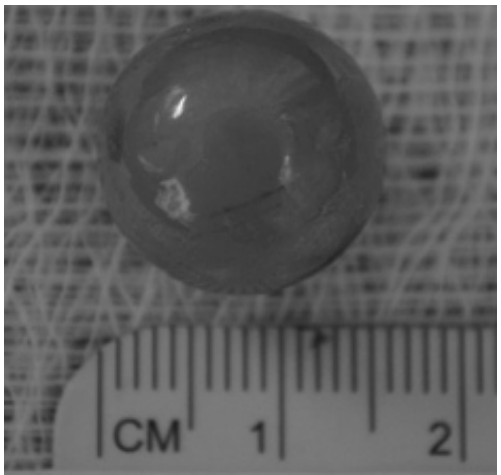

Figure 4. The $1.7 \times 1.7 \mathrm{~cm}$ glass marble

\section{DISCUSSION}

It is very common to see a foreign body in the nasal cavity or in the external ear canal but seeing it in unlikely places like the sphenoid sinus is such a surprise. Many factors need to be considered in the decision to extract it. One factor to consider is the approach to the sphenoid sinus. There are two different approaches to the sphenoid sinus: external and internal. The external, trans-ethmoidal approach involves subperiosteal elevation and ethmoidectomy. ${ }^{2}$ Internal approaches such as the transseptal and trans-nasal are less-invasive ways to access the sphenoid sinus. Because of ease in access, minimal damage to surrounding mucosa and good exposure, the trans-nasal approach was used. Whatever approach the surgeon chooses, it is important to be familiar with the surgical anatomy to prevent unwanted complications. Creativity also played a role in this procedure and quick thinking was needed since the foreign body was a round object and extracting it from such a limited space with utmost care using makeshift instrumentsmwas critically challenging.

Sincerely,

Alvin B. Javierto, MD

Josefino G. Hernandez, MD

Rodante A. Roldan, MD

Department of Otolaryngology

Head and Neck Surgery

Rizal Medical Center 